\title{
COMB: Cell based Orientation aware MANET Broadcast MAC layer
}

\author{
Cristina Rico García*, Andreas Lehner*, Thomas Strang* \\ ${ }^{*}$ German Aerospace Center \\ Institute of Communications and Navigation \\ 82234 Wessling \\ Germany \\ Email: \{cristina.ricogarcia, andreas.lehner, thomas.strang\}@dlr.de
}

\begin{abstract}
The design of a collision avoidance system for trains implies the design of a MAC layer for their specific requirements. It should be efficient, reliable, use broadcast and support wireless mobile ad hoc networks (MANETs) with high user speeds. Therefore we are using awareness techniques, which allow a certain channel assignment, despite the absence of infrastructure. This paper presents a new MAC layer protocol designed for broadcast MANETs called COMB (Cell-based Orientation-aware MANET Broadcast). In principle, COMB allows the realization of a collision free transmission, high speed is supported and no handshake is required. COMB is based on localization aware cross layer dimensioned CDMA cells, and uses the SOTDMA protocol as intra cell scheme, while the inter cell scheme relies on direction and speed awareness.
\end{abstract}

\section{INTRODUCTION}

The number of accidents in traffic let grow the number of collision avoidance systems in the past years. In air, maritime and road traffic there were presented mechanisms that prevent collisions by communication of traffic participants avoiding more or less any infrastructure.

In railroad traffic on the other side, current approaches mainly use infrastructure - and focussing therefore only on main railways, as infrastructure is expensive. To overcome these problems we are working towards a system that is avoiding train collisions by broadcast communication of trains, taking into account parts of the present traffic situation, in order to be able to provide the necessary reliability.

Though the communication needs of trains are different from those in all other kinds of traffic. The channel model, the physical layer and the medium access control has to be adapted to the present needs. Railroad transportation is challenging. It presents a large speed combined with a demanding propagation channel and high density of trains at some points. This paper will focus on the MAC layer. In our case the MAC layer should be broadcast, there is in no case addressed communication. We present a new, reliable MAC protocol for Broadcast VANETs called COMB that is designed to perfectly meet railway requirements.

The remainder of this paper is organized as follows: In section 2, we discuss the requirements of our system regarding its MAC layer and compare them with the approaches offered by the state of the art. Following that we will present our new COMB protocol for Medium access control before section 4 will show our simulation results. Finally, section 5 provides the summary of our efforts in that field.

\section{REQuirements AND StATE OF the ART}

A plethora of MAC protocols have been proposed in the literature so far. Obviously however, different usage scenarios imply different threats and challenges. According to these, we can see an evolution in the protocols. Here we want to distinguish four major steps in detail.

\section{A. Wireless MAC}

The core feature of an ad hoc network is to provide communication services without any infrastructure or centralized access point. Therefore, there is no base station to coordinate packet transmissions. Since channel resources are limited, transmissions will interfere with these neighbors that also have packets to transmit in the same channel. Consequently, the MAC (Medium Access Control) layer must be accomplished in a distributed way.

\section{B. Mobile ad hoc network (MANET) MAC}

Moreover, ad-hoc networks allow stations to move which introduces more complexity, as it causes permanent network changes. This may significantly impact on the MAC layer's performance specially as in our case when the speed is high.

The simplest protocol for MAC layers that can be used is the well known ALOHA protocol [1] where no control is used, however due to its low throughput it is only applicable in low density ad-hoc networks. Another of the earliest mechanism adopted was the CSMA (Carrier Sense Medium Access) protocol [2]. Nonetheless, it introduces the hidden terminal and exposed terminal problem. Thus, a variety of more complex MAC protocols have been proposed for mobile ad hoc networks (MANETs). Some of these general protocols include the Multiple Access with Collision Avoidance (MACA) [3], Media Access Protocol for Wireless LAN's (MACAW) [4], Floor Acquisition Multiple Access (FAMA) [5] and the MAC protocol of IEEE 802.11 [6].

\section{Broadcast MANET MAC}

Unfortunately, these protocols are not suited for broadcast MANETs, as the required handshake cannot be performed, 
if we don't have a specified communication partner (CSMA, MACA, MACAW,...) or performance is too low (Aloha).

Beside these, many MAC protocols for broadcast MANETs have been proposed in the literature. They can be classified in two groups:

- Improved versions of the widely accepted IEEE 802.11 MAC protocols. They include BSMA [8], BMW [9], BMMM [10], LAMM [10]. Unfortunately, the above listed broadcast MANET MAC protocols are not suited for complex dynamic and very extended MANETs, characterized by high sender density and high speed, because they use handshake techniques in order to recognize possible packet collisions and retransmit again the messages. Though handshake is not possible if you don't know your communication partner as in our MANETs.

- Innovative MAC protocols related to TDMA, FDMA or CDMA. Random access protocols such as CSMA/CA have proven not to be an efficient solution. Therefore, starting from the basic TDMA or CDMA is an important research topic. In this group we can find ADHOCMAC [11], Five-Phase Reservation Protocol (FPRP) [7], Self Organized Time Division Multiple Access (SOTDMA) [12], CATA [13], ABROAD [14], RBRP [15] and SNDR [16]. These protocols usually assume quite static networks and prior knowledge of the number of receivers, which is not given. Furthermore they don't provide a solution for the hidden (see Figure 1) and exposed terminal problem.

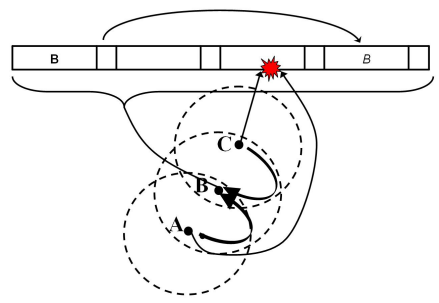

Fig. 1. Hidden terminal problem: A and C try to access the medium in the same channel because they can not "see" each other.

An example of extended and highly dynamical MANETs using broadcast are collision avoidance systems for transportation, e.g. road (Car2Car), maritime (AIS) , air (ADS-B), railway (RCAS). These systems might be deployed in such a large area as the whole world. These systems broadcast periodically data inferred from the on-board Global Navigation Satellite System (GNSS). ADS-B utilizes a protocol based on Aloha. However, as the speed and the senders' density increases, the number of packet collisions due to the hidden terminal problem and collisions in contention time are more critical. Car2Car uses a protocol based on IEEE 802.11, whose problems have been already discussed. AIS MAC protocol is Self Organized Time Division Multiple Access (SOTDMA) a kind of split channel reservation multiple access (SRMA).

SRMA protocols have two channels, a control channel and a data channel. In the control channel, the senders compete for the data channel. SRMA protocols can be implemented in many ways: the control and data channel can be differentiated in frequency, code or time. As a result, in the absence of the hidden terminal problem, collisions can only occur in the control channel during the contention time.

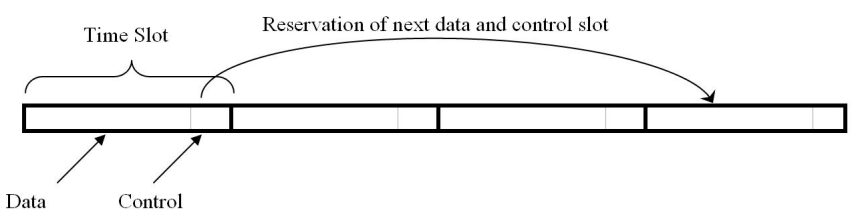

Fig. 2. SOTDMA MAC protocol.

In order to additionally decrease the collision probability in the control channel, a sender reserves not only a data channel but another control channel for the estimated next transmission. Since this scheme is developed in time, it is specially suited for TDMA schemes. Consequently, assuming no hidden terminal problem, once a terminal has entered the network and has obtained successfully data and next control channel, no more collisions occur among the terminals within the network.

In SOTDMA control channel and data channel are TDMA, as well as the channels within the control and data channel. They are combined in the following way: the channel is divided into slots, in each slot there is a sub-slot for data and another one for control data. A "slot frame" is the minimum number of slots between two consecutive messages of a node. When a new sender comes into the network, it searches a free slot. In the free slot it sends the data and in the control sub-slot it reserves the next data and control slot as shown in Figure 2. Therefore, each node in a network knows in which slots the nodes in its range are planing to transmit, and thus, can infer which slots are free.

\section{Awareness for MAC}

Since GNSS is very oft present in high dynamic MANETS, the information given by the GNSS system (location, direction, speed, and precise timing) can be utilized by the MAC layer. Thus, our below presented MAC layer protocol COMB relies on location awareness, a subspace of situation awareness.

A number of MAC protocols utilizing position information have been proposed, like GRID [17] and TPCPC [18]. However, these protocols are focused on unicast communication. Moreover, they rely on a handshake protocol and therefore there might be collisions during the contention.

\section{E. Summary}

This overview shows, that there still is a need for a MAC protocol allowing for mobility, broadcast and situation awareness and handling all related problems. COMB does not only rely on position, but on speed, direction, and time awareness in order to allow a robust protocol. It does not either use any handshake protocol in order to recognize and recover problems 
caused by packet collisions simply because collisions do not occur in the frequency domain.

\section{THE COMB CONCEPT}

As we have seen the first problem to solve is the hidden terminal problem. We will propose in this section a solution for this and consequent problems before we finally present the single steps of our COMB protocol.

\section{A. Solution for the hidden terminal problem}

The hidden terminal problem is produced when two nodes, $\mathrm{A}$ and $\mathrm{C}$, are not in the range of each other, but are both in the range of another node B as can be seen in Figure 1. In SOTDMA B would "see" the slots reserved by A and C. However, since $\mathrm{A}$ and $\mathrm{C}$ cannot see each other, they might try to access the same slot and collide. Nonetheless, if the nodes A and $\mathrm{C}$ send their messages with different CDMA codes, node $\mathrm{B}$ can receive both messages at the same time. Thereby it is only necessary to assign distinct codes to "far-away" nodes, as the hidden terminal problem doesn't arrise, if all nodes see each other.

In order to accomplish this idea, the world map is divided into virtual hexagonal cells with different message codes. The dimension, i.e. the diameter, of the cell should be in the order of the range of the nodes, as seen in Figure 3. This way, every node in any position inside a cell is able to receive signals from the other nodes of the same cell. Thus, the SOTDMA structure is seen by all the nodes avoiding this way possible collisions. Furthermore, since messages from other cells are received with another (orthogonal) code, there are no collisions due to the hidden terminal problem as observed in Figure 4. The CDMA code with which a node has to transmit is inferred according to its location in a cell calculated from its GNSS system.

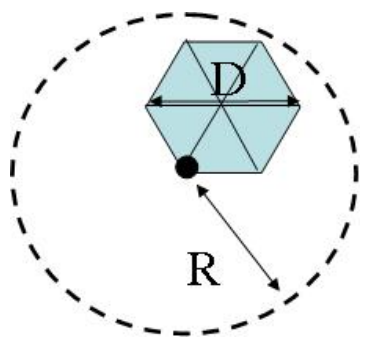

Fig. 3. The cell dimension is related to the range (see Eq. 1).

In order to limit the number of necessary orthogonal codes, we want to reuse codes. With sufficient guard spaces between codes that share the same code, we still can avoid the hidden terminal problem. Figure 5 shows that three cells are the minimum for that, considering the range of trains in the corners of a cell. Consequently, twelve codes as seen in Figure 6 constitute a network free of interference and hidden terminal problem.

\section{B. Solution for contending nodes}

Another problem arises when the nodes move, as they have to contend for a new slot in a new cell. When more than one

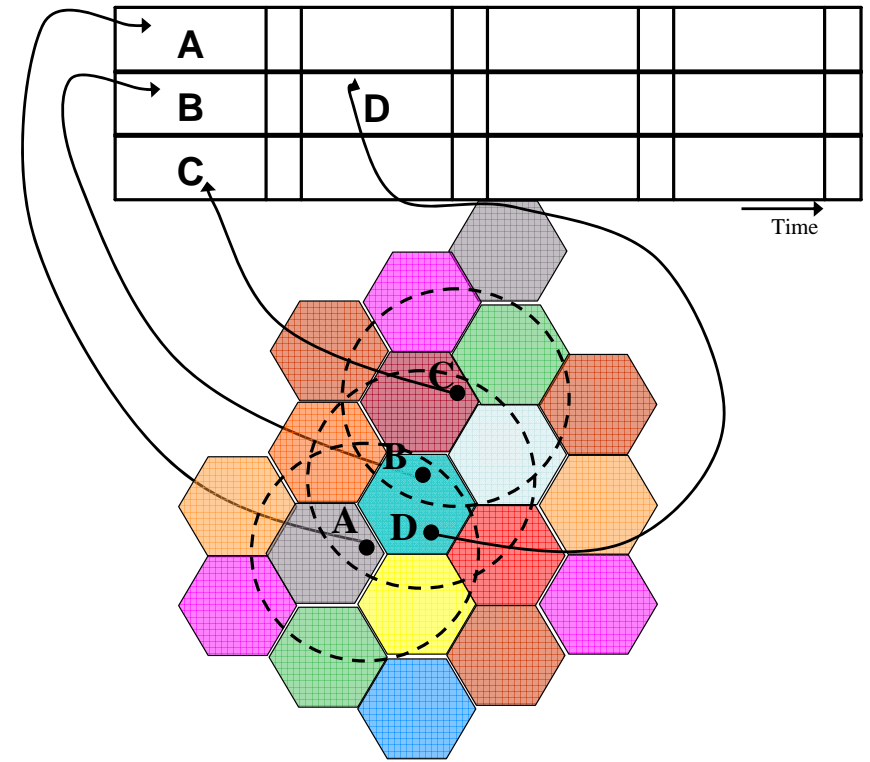

Fig. 4. Different colors mean different codes.

$\mathrm{A}, \mathrm{B}$ and $\mathrm{C}$ try to access the same time slot, but with different codes. Therefore, there is no collision. D and $\mathrm{B}$ are in range of each other and send with the same code. Consequently, they choose different time slots and there is no collision, either.

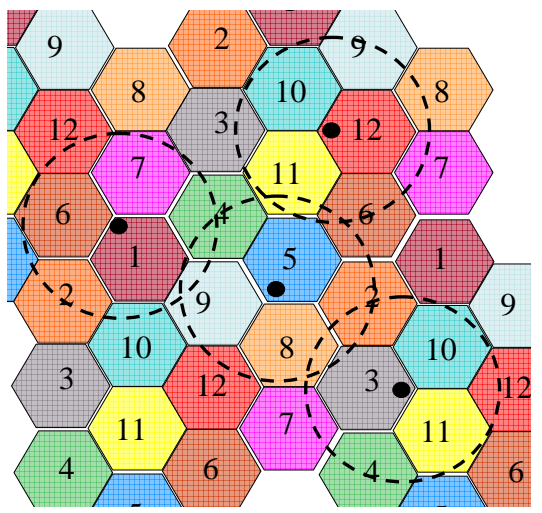

Fig. 5. When there are three cells between two cells that share the same code, the hidden terminal problems does not occur.

node crosses to the same cell, they may try to access the same slot and a collision occurs as observed in Figure 7.

Due to the fact that the range of the nodes is slightly bigger than the cell dimensions, the nodes close to the border of other cells are able to receive all the transmitted signals from that cell, and therefore know its SOTDMA structure. With their speed and direction (e.g. from a GNSS system) the nodes know with antelation to which cell they are about to cross. While nodes usually reserve a slot for their next message in the SOTDMA slot structure of their current cell, they have to reserve the next slot in the SOTDMA structure of the target cell, if they will send their next message already in a different cell. I.e. they make the reservation indicating that the next code they will use is the one of the target cell. Hence several nodes coming from a same cell "1", will never produce a packet 


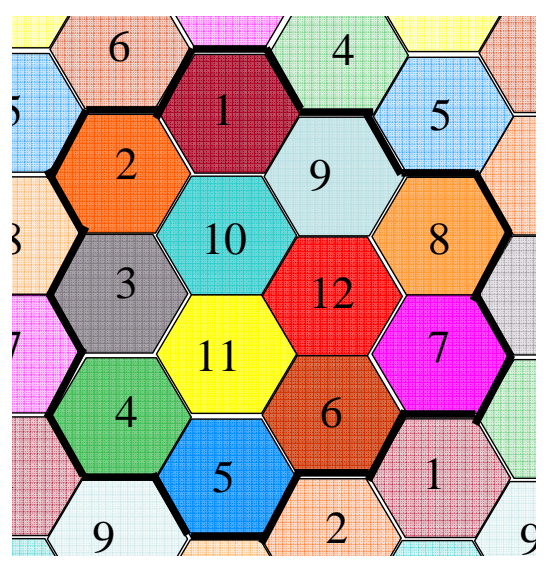

Fig. 6. The basic cell pattern with 12 different orthogonal CDMA codes. Ordering of the codes is arbitrary, but has to be equivalent for all neighboring cell groups.

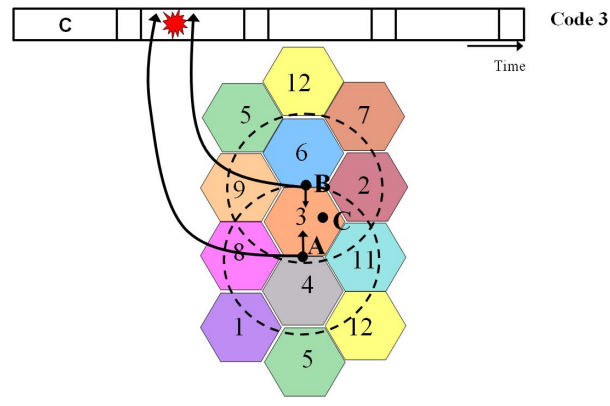

Fig. 7. Collision of contending nodes: A and B move into a new cell and must change their CDMA codes. However, they try to access the channel in the same time slot and collide.

collision due to contention when they cross to cell "2".

However, nodes crossing from different cells to a same one may collide. Since one cell is surrounded by six cells, there might be a maximum of six nodes contending for a same slot. In order to solve this problem, we will define a "cell crossing priority". The cell situated in the north of the target cell has priority 1 , the clockwise next has 2 and so on until priority 6 . Like that, a crossing train with priority $i$ will reserve the $i$ th free slot of the next slot frame in the target cell. Therefore, the nodes crossing to a target cell should listen to the slot frame of the target cell and the surrounding cells.

\section{Cross layer characteristic of COMB}

An important issue of COMB is the cross layer dependency. The dimension of the cell should be smaller than the range of a train, which is controlled by the physical layer. In order to minimize problems caused by the variability of the range, we should assure the existence of three cells with different codes between two cell sharing the same code. In this case twelve orthogonal codes are necessary as seen in Figure 6.

The ideal cell dimension $D$ is

$$
D=R-2 \cdot F
$$

where, $R$ is the minimum range and $F$ is the distance of a slot frame (one is necessary to observe the slot frame of the

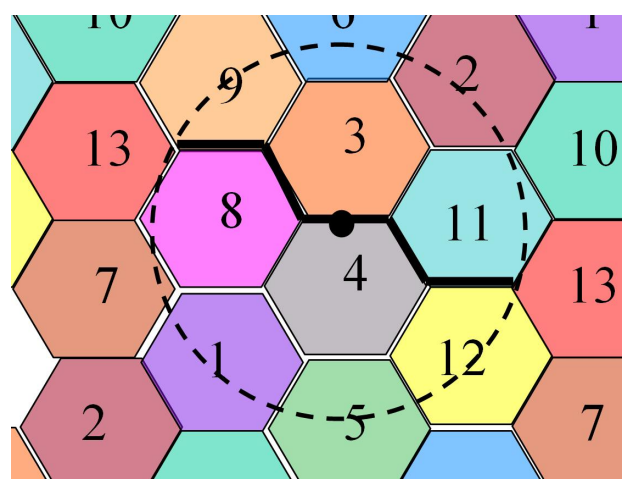

Fig. 8. The maximum range is given for a node located in the middle of the shortest distance between two co-channel cells.

target cell, and a second one to make the reservation). $F$ can be calculated as $F=v \cdot t$, where $v$ is the maximum speed of the node and $t$ is the minimum time between two consecutive messages of a node. E.g. if the nodes have a range of $7 \mathrm{~km}$, the messages are sent each second, and it is moving at 300 $\mathrm{km} / \mathrm{h}$, then in order to receive two slot frames before crossing to the new cell $2 \cdot F=(300 \cdot 1000) \cdot \frac{2}{60 \cdot 60}=0,167 \mathrm{~m}$

Considering the cell size given by the minimum range, then the maximum range would be delimited by the maximum range where no hidden terminal problem is present. This is given for a node located in the middle of the shortest distance between two cells sharing the same code, as seen in Figure 8. This node would have the borders of its range in the borders of the cells with same code.

Therefore, the range has a certain margin where it can move and still no collision is produced. However in case the necessary margin would be bigger, more codes can be added to the system.

\section{COMB protocol}

1) Divide the map in hexagonal cells where its dimension is given by equation (1).

2) Give each cell a code of a group of twelve orthogonal CDMA codes in such a way that there are three cells between two cells with same code.

3) Inside a cell the messages are codified with the corresponding cell code.

4) Inside a cell the messages are sent according the SOTDMA protocol. The time is divided in "slot frames" where the frame duration is the minimum time between two consecutive messages coming from a same node.

5) The nodes observe the "slot frames" codified with its cell code. The nodes send their messages in their already reserved slots and reserve a free slot in a next "slot frame".

6) When a node is about to cross a cell border, it must observe the "slot frame" of the target cell and reserve one of the first six free slots according to its current's cell priority.

7) The cell priority is given by the position of the current cell with respect to the target cell. The highest priority 
corresponds to the cell in the north.

\section{Preliminary Simulation Results}

For the simulations we use an event driven programm. The packets are 200 bits long, and every node sends a packet periodically once per second. The nodes are moving at 350 $\mathrm{km} / \mathrm{h}$ in randomly distributed linear paths in a $100 \mathrm{~km}^{2}$ area. The minimum propagation range is $5 \mathrm{~km}$ and thus, the diameter of the cells is more than $4.8 \mathrm{~km}$. The SOTDMA frame has $80 \mathrm{slot} / \mathrm{s}$ and the codes have a length of 128 chips. A message has 170 data bits the necessary overhead for the COMB protocol are in this case 9 bits, three to reserve the code and six to reserve the next slot.

In an ideal scenario, i.e., the cross correlation characteristics of the codes are perfect, and the channel is ideal, no collisions occurred during the simulations.

Theoretically, a collision free broadcast of information is achievable using the COMB strategy. In practice, the performance is degraded due to the near-far problem in CDMA based systems if the required transmission range increases. A worst case situation for ground based transportation systems are the requirements in railway transport.

To allow secure breaking in case of a collision threat, a range of $5 \mathrm{~km}$ is required. Moreover propagation conditions in regional railway network can vary in a wide range. Therefore, On a second step we simulated the near far effect, including a restrictive suburban model for the path loss [19], the used frequency band was $400 \mathrm{MHz}$ and the antenna height 4,8 $\mathrm{m}$. The interference threshold was set to $16 \mathrm{~dB}$. Under these worst case situation the following plot shows the results for the proposed COMB MAC protocol, which shows that the collision rate remains low under such demanding conditions specially in comparison with the SOTDMA protocol.

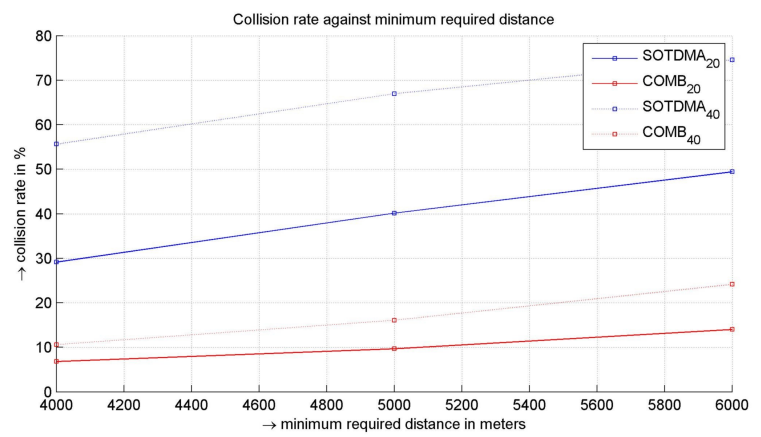

Fig. 9. Collision rate of the COMB and the SOTDMA protocol for $20 \%$ and $40 \%$ load.

\section{CONCLUSION AND OUTLOOK}

In this paper we have presented COMB, a new MAC layer for broadcast MANETs. COMB divides the network in cells characterized by one CDMA code while within each cell an SRMA protocol is used. Our protocol cooperates with the physical layer in order to control the range. The protocol is orientation aware and using GNSS data. Positioning is necessary in order to recognize in which cell the vehicle is and which CDMA code should be utilized. Speed and direction information are used when crossing to another cell.

With this approach, we can avoid the hidden and exposed terminal problem as well and collisions due to contention neither in control nor in data channels. The necessary overhead is minimum and since only 12 CDMA orthogonal codes are necessary, it is practically realizable. Furthermore, it is easily scalable and the terminals may move as fast as required.

In future work we will optimize the number of codes depending on the required propagation range and we will study the effect of the dimensioning and sectorization of the cells in presence of hot spots.

\section{REFERENCES}

[1] N. Abramson, "The ALOHA System-Another alternative fo rcomputer communications", 1970 Fall Joint Comput. Conf., AFIPS Press, vol37, pp. 281-285, 1970

[2] John Jubin and Janet D. Tornow, "The DARPA Packet Radio Network Protocols", Proceedings of the IEEE, January 1987.

[3] P. Karn, "MACA-A New Channel Access Method for Packet Radio", Amateur radio 9th Computer Networking Conference, ARRL, 1990.

[4] R.Bargrodia, A. Demers, S.Shenker, and L. Zhang, "MACAW: A Media Access Protocol for Wireless LAN's" ACM SIGCOMM, 1994.

[5] C. Fullmer and J.J Garcia-Luna-Aceves, "Floor Acquisition Multiple Access (FAMA) for packet radio networks", ACM SIGCOMM, Cambridge, October 1995.

[6] Editors of IEEE 802,11, Wireless LAN Medium Access Control (MAC and Physical Layer (PHY) specifications, Draft Standard IEEE 802.11, 1997.

[7] Chenxi Zhu and M. Scott Corson, "A Five-Phase Reservation Protocol (FPRP) for Mobile Ad-Hoc Networks" Proc. INFORCOM'98, 1998.

[8] K. Tang and M. Gerla, "Random access MAC for efficient broadcast support in ad hoc networks", WCNC, pp. 454-459, September 2000.

[9] K. Tang and M. Gerla,"MAC reliable broadcast in ad hoc networks", Proceedings of the IEEE Military Communications Conference (MILCOM), pp. 1008-1013, October 2001.

[10] M. T. Sun et al.,"Reliable MAC layer multicast in IEEE 802.11 wireless networks", ICCP, pp. 527-536, August 2002.

[11] F. Borgonovo et al. "ADHOC MAC: a new, flexible and reliable MAC architecture for ad-hoc networks" IEEE Wireless Communications and Networking Vol. 2, WCNC 2003.

[12] Høakam Lans, Saltsjöbaden, "Position Indicating System" United States Patent, Patent Number: 5506587, Apr. 9, 1996.

[13] Z. Tang and J.J. Garcia-Luna-Aceves, "A protocol for topologydependent transmission scheduling in wireles networks" Proceedings of IEEE WCNC'99, pp. 1333-1337, September 1999.

[14] I. Chlamtac et al.,"An adaptive medium access contorl (mac) protocol for reliable broadcast in wireless networks" Proceedings of IEEE ICC'00, June 2000.

[15] Mahesh K. Marina et al.,"A Robust Broadcast Reservation Protocol for Mobile Ad Hoc Networks" Proceedings of the IEEE International Conference on Communications (ICC), vol. 1, pp. 878-885, June 2001.

[16] Zhijun Cai and Mi Lu, "SNDR: A New Medium Access Control for Multi-channel Ad Hoc Networks", Proceedings of the Vehicular Technology Conference (VTC), Tokyo 2000.

[17] Yu-Chee Tseng et al.,'Dynamic channel allocation with location awareness for multi-hop mobile ad hoc neworks" Computer Communications, Elsevier, 25, pp. 676-688, 2002.

[18] Lili Zhang, Boon-Hee Soong, Wendong Xiao, "A New Multi-Channel MAC Protocol for Ad Hoc Networks Based on Two-phase Coding with Power Control (TPCPC)",ICICS-PCM 2003, Singapore, 15-18 December 2003.

[19] Cristina Rico Garca, Andreas Lehner, Thomas Strang, Korbinian Frank "Channel Model for Train to Train Communication using the $400 \mathrm{MHz}$ Band" 67th Vehicular Technology Conference (VTC2008-Spring), Marina Bay (Singapore), 2008 